2. There was one ease of actinomycosis in measures in the treatment of abscess of the lung. which the diagnosis was made by Dr. William H. There were nine deaths, eighteen recoveries, and Smith in conjunction with Dr. James H. eleven cases were practically well without Wright, after painstaking study. This case of cough or sputum.

actinomycosis was cured by surgical operation. It suggests that actinomycosis of the lung must be recognized in its initial stage and vigorously attacked by surgery, for only in this way is there a great likelihood of euring this very fatal disease.

3. Attention is attracted to lung abscess following tonsillectorny. There have been several cases in my experience. There is one case in this series which followed operation upon the nasal septum. The inhalation of foreign material may have been the starting point in this case. The employment of intratracheal anesthesia with gauze packing of the pharynx may be wise in these cases.

4. From certain cases in this series it is suggested that the surgeon should be alert to the occurrence of lung abscess associated with embolism following ordinary surgical operations. A patient suddenly suffering severe pain in the chest following surgical operation associated with a rise in temperature and pulmonary signs should be very carefully studied lest such signs may mean the beginning of pulmonary abscess. Early operative interference upon the lung in such cases may not only preclude the possibility of the extension of a pulmonary lesion, but may save the life of the individual.

5. An increasing number of intra-abdominal infections are associated directly with an infection through the diaphragm of the diaphragmatic pleura, and then of the lung with pulmonary abscess. These cases of sub-diaphraginatic origin must be attacked early to prevent subsequent damage intrathoracically.

6. The x-ray is of great value in the diagnosis of lung abscess. The thorough roentgenological examination necessitates fluoroscopic observations, stereoscopic plates, and both anterior and posterior views. If there is reason to suspect fluid in the chest, the examination should be made in the upright, as well as in the horizontal position. The exact position of the heart, the position and excursion of the diaphragm, and the clearness of the costo-diaphragmatic angle, are facts which help in the interpretation of the $\mathrm{x}$-ray plates.

The recognition of the presence or absence of tuberculosis, of a diffuse or bilateral bronchiectasis, of syphilis of the lung, will assist in the interpretation of the plates. Centrally seated abscesses, almost impossible of determination by ordinary physical signs, may be accurately localized by the x-ray.

'The $\mathrm{x}$-ray is of assistance in the interpretation of physical signs in cases of suspected lung aliseess and bronchiectasis, and may be of assistance in the exact localization of the abscess.

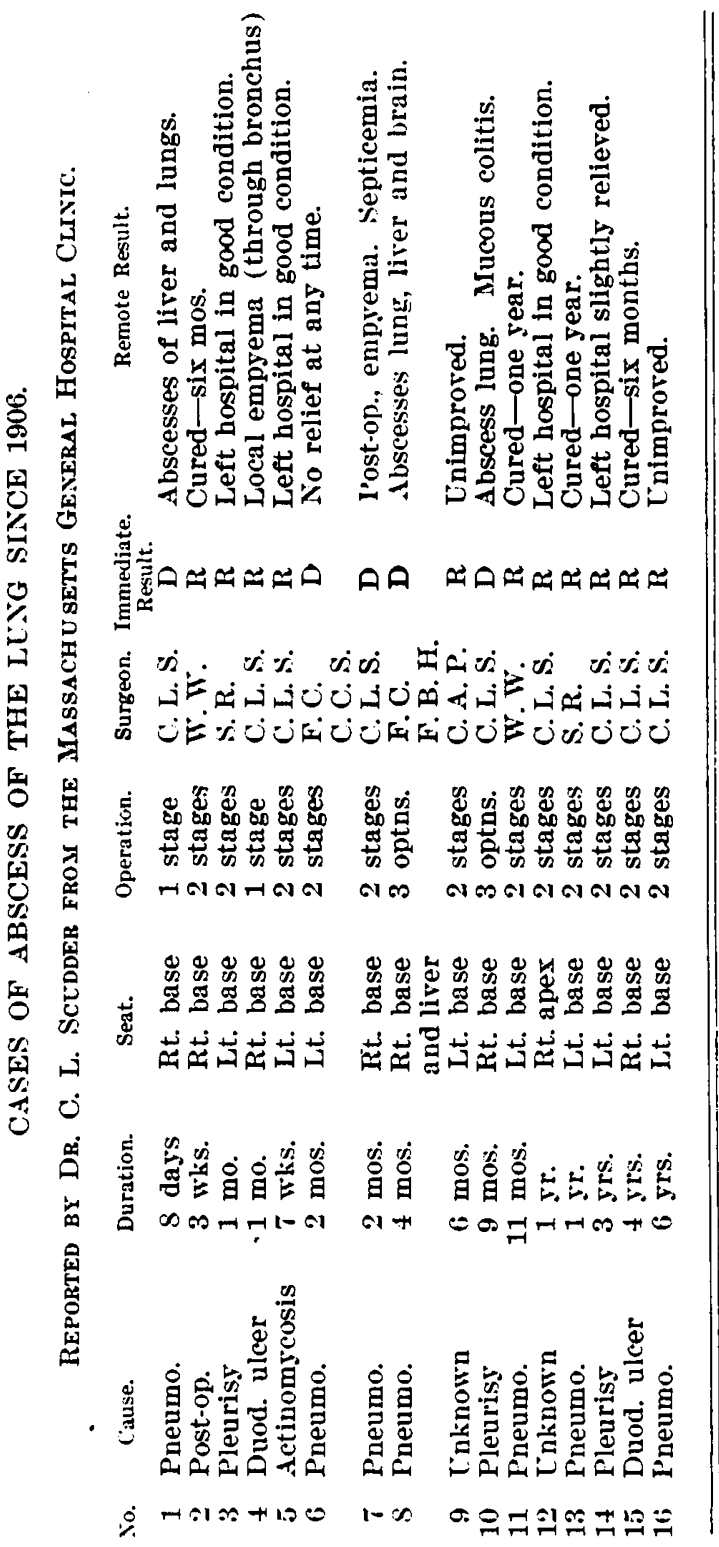

\title{
OBSERVATIONS ON THE BEHAVIOR OF NEOSALVARSAN.
}

\section{By W. J. McGurn, M.D., Boston.}

BEFORE entering into a general discussion of this subject, it is well to recall that ample proof has been furnished that severe illness and death sometimes follow close upon the intravenous ad. ministration of salvarsan and its derivative neosalvarsan. While it appears that autopsy offers conclusive evidence that death in many eases

7. Finally, $I$ believe that this total series of was due to some external agent introduced at 27 eases demonstrates the efficacy of surgical the time of injection, it remains to be proven 
whether either of these agents, unmodified and in perfect solution, may result in a fatality.

Anyone with even a limited experience with neosalvarsan is, of course, familiar with Ehrlich's warning that solutions of neosalvarsan should not be warmed, and to be safe the temperature must be kept below $70^{\circ} \mathrm{F}$. Also, that immediate injection after its preparation is imperative. To the average mind the question promptly arises: Why then is it not dangerous to subject the solution to a temperature of $98.6^{\circ}$ F. by introducing it into the blood stream? The answer most often given is a speculative one based upon theory rather than observation. The truth of the matter, however, is that neosalvarsan is unaffected by a very wide range of temperature when the drug, or its solutions, is not in contiguity with free or loosely combined oxygen.

This deduction was obtained after observing the behavior of two hundred specimens of the drug, subjected to various experimental tests, the results of which seem to be conclusive.

The chemical change taking place in solutions of neosalvarsan is due to the process of oxidization, the rapidity of which depends upon the area of surface exposure; and to the temperature of the air to which it is exposed rather than upon the temperature of the solution itself. Solutions of neosalvarsan prepared from ampuls that have been subjected for several hours to a temperature of $100^{\circ} \mathrm{F}$. do not differ from those not so treated.

Oxidization always begins at the top of the solution where it is in contact with air. The first evidence of this process is seen in a whitish opaque zone overlying the transparent solution, which is not readily detected until the zone of opacity assumes the depth of one-eighth to onefourth inch, unless the surface of the solution is elevated to the visual level and inspection made by transmitted light. Even then slight agitation of the mixture will obscure the presence of oxidization products, so that we are dealing at once with a mixture of unknown toxicity instead of a solution of neosalvarsan.

Specimen tubes of fresh prepared solution placed on ice oxidize more slowly than do similar tubes placed in hot water, but the difference in time of decomposition is not so great as might with reason be expected; while reverse results are obtained when the exposed surface of the iced solution is subjected to a draught of warm air, and that of the warm solution treated with a current of air chilled to $50^{\circ} \mathrm{F}$. This experiment tends to indicate that a low room temperature is of even greater importance to the safe handling of neosalvarsan than is a low temperature of the solution itself.

When solutions of neosalvarsan are prepared in shallow receptacles of great surface area, oxidization progresses rapidly and the process is usually well advanced in three to five minutes; while containers of small surface exposure permit of much slower deterioration of the content, decomposition being further retarded, but not prevented, by maintaining the room temperature at or below $65^{\circ} \mathrm{F}$., also by keeping the solution closely covered.

'Test tubes of small diameter, when filled with solution and tightly corked, usually show degenerative changes in about one hour, which change becomes complete in from one day to one week. An air bubble of pin-head size is capable and certain of bringing about a complete chemical change within a few days; while tubes sealed hermetically, after the complete expulsion of air, keep indefinitely even when subjected to a temperature of $200^{\circ} \mathrm{F}$.

To insure a permanent solution, however, it is necessary to exclude not only direct sunlight, but diffused daylight as well. Specimens so prepared and exposed to sunlight do not give evidence of physical or chemical changes for a period of from three to ten weeks, and when subjected to diffused light only, they remain in solution a much longer time, usually for three or four months.

Comparative tests show that saline solution prepared with our metropolitan tap water boiled, cooled and filtered through a fine porcelain tube is not inferior to fresh distilled water for intravenous administration of neosalvarsan.

Solutions prepared by using $8 \mathrm{cc}$. of a $0.4 \%$ salt solution to each $0.1 \mathrm{gm}$. of neosalvarsan are equally safe and hold up much better than those diluted to a greater volume of the saline, while concentrated solutions offer even a higher degree of stability. Neosalvarsan when diluted further than advised by the Ehrlich laboratory is likely to become suddenly suspended and a faint opacity appear throughout the liquid.

There can be no doubt that neosalvarsan is prepared and sealed with very accurate precautionary measures, but the assumption would be too great to suppose that thousands of these ampuls could be dispensed from any laboratory and each present exact uniformity of conduct when subjected to various thermal, chemical and clinical observations.

While most ampuls of neosalvarsan are exactly similar in their behavior when exposed to air, it is also true that occasionally one is encountered which for an unknown cause appears higher in color, dissolves with some difficulty and oxidizes much more rapidly than others. Occasionally I have found an ampul where extensive oxidization of the neosalvarsan took place within ten minutes after being dissolved, while with other ampuls but slight evidence of deterioration could be detected at a period of two hours after the solution was prepared. In fact such a variation of conduct was observed by me where every environment remained the same throughout the day, so far as could be determined.

Oxidization of neosalvarsan when exposed is not hastened by the presence in the air of such chemical substances as alcohol, ether, chloro- 
form, carbolic acid or illuminating gas, while exposure to an atmosphere where liquid oxygen has recently been used, will cause sudden and complete precipitation of the drug. Minute traces of mercuric bichloride or wood alcohol are rapid in their destructive action upon aeosalvarsan, while one drop of hydrogen peroxide is eapable of sending it down with a proverbial thud, ammonia presenting another form of incompatibility.

Careful examination by transmitted light would indicate that neosalvarsan when dissolved in $0.4 \%$ chemically pure sodium chlorid solution fulfills every requirement of a "pcrfect chemical solution." This belief is disproven, however, by passing the mixture through a white porcelain filter capable of negotiating the complete ampul content to the time of three minutes. Solutions so tested invariably leave a residue, the presence of which is brought out very clearly by adding one or two drops of peroxide hydrogen, or by swabbing the exterior surface of the filter with liquid oxygen. The amount of the residue is increased by delaying filtration and by diluting beyond $8 \mathrm{cc}$. of $\mathrm{NaCl}$ solution to each 0.1 gram of neo. When elinically employed the porcelain filter is capable of preventing any insoluble or oxidized portion of the drug from entering the vein and is always a safeguard against an impure filtrate.

After a fairly extensive experience with neosalvarsan I am convinced that when accurately handled it is both safe and dependable; that in preparing and administering this agent a cool room with perfect illumination is of equal importance with strict asepsis; that the beginning of oxidization of neosalvarsan is simultaneous with its exposure to air; that "immediate injection" should be interpreted as forbidding a delay of more than ten minutes from the time of opening the ampul until the injection is completed, which time can easily be reduced by half; also, that the use of therapeutic agents containing ammonium carbonate or mercury should be withheld or suspended for a period of fortyeight hours preceding and for several days following the intravenous administration of either new or old salvarsan.

\section{OUliniral Bppartntent.}

\section{SYPIIILITIC MULTIPLE SCLEROSIS DI- AGNOSED CLINICALLY IN SPITE OF NEGA'TIVE I,ABORATORY TESTS.}

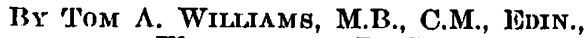
Wasingaton, D. C.,

Neurologist to Epiphany Dispcnsary, Corresponding Member, Paris Neurologic Society.

A WOMAN aged thirty-nine was sent to me from New York state because of what had been diac nosed by several neurologists as multiple sclerosis. Her physician wrote asking if I could suggest some treatment or perhaps inodify the diagnosis.

The patient declured that she had been intensely nervous since the birth of her boy eight years ago; since two and one-half years this had consisted of palpitation and a feeling of dread, great unsteadinoss of the hands, voice and gait. In addition there was marked incontinence of urine and extreme obstipation with oceasional incontinence. She was dizzy when tired, and the eyes were blurred.

Exarnination showed great exaggeration of the tendon reflexes, but, no corresponding diminution of the cutaneous reflexes, and the great toes did not extend upon stroking the sole of the foot nor by other stimuli used to provolse that reflex. But that there was some interference with the pyramidal system was shown by absence of reflex flexion of the toes, all of them remaining immobile to the stimuli of reflectivity.

The absence of the sign of Babinski in a patient whose illness was of such gravity and durntion made me snspeet that insular sclerosis might not be present after all; and on proceeding to the examination of the eyes, my doubts received further support; for no nystagmus occurred even during rapid intentional movements; and the optic papilla showed no atrophy, although slightly pale. The absence of three signs of such importance compelled me to contradict the previous dingnosis, in spite of the presence of intention tremor, scanning speech, impaired diadocokinesis, a slight moria, such as often occurs in insular sclerosis, and a diminution of vibration sense in the legs.

But it is pretty well known to neurologists that the symptoms of insular sclerosis may be simulated. even to a greater degree than was shown by this patient, when there is a diffuse chronic inflammation of the nervous system produced by the treponema pallidum. This I suspected to be the condition of this patient.

Accordingly lumbar puncture was done on February 13 , when 30 c.c. of fluid very rapidly came and Dr. Nichols reported that there were 7 lymphocytes per c.m. This slight lymphocytosis still left us in doubt, for an increase in cells has been reported in cases of insular sclerosis.

However, I put the case to the patient, who decided to permit the experiment. So I injected a full dose of salvarsan into a vein on February 22. This rendered her almost helpless for about two dnys. Then she began to handle herself much better, and on the 28th she also felt minch better and the motility still further improved. The reflexes and sensibility were as before, but the intention tremor had greatly diminished.

So on March 7 the spinal fluid was acain examined, and a luetin test was begun by Dr. C. A. Simpson. The eclls lad diminished to $5 \mathrm{cem}$. This puncture caused grent pain. headache and nausen. $\Lambda$ fter this clcared up, salvarsan was again given on March 15. Within 24 hours her legs gave way twice, but a great improvement again followed in walk and speech. The intention tremor disappeared, and her sleep became reatful. 'The Wassermann reaction remained negative as before; but a papulc rapidly formed where luetin had heen injected, and when I saw her five days after the salvarsan, a broken pustule was present which Dr. Simpson pronounced a luetin reaction. 